\title{
Storm-time ionization enhancements at the topside low-latitude ionosphere
}

\author{
A. Dmitriev ${ }^{1,2}$ and H.-C. Yeh ${ }^{1}$ \\ ${ }^{1}$ Institute of Space Sciences, National Central University, Chung-Li, Taiwan \\ ${ }^{2}$ Skobeltsyn Institute of Nuclear Physics Moscow State University, Moscow, Russia \\ Received: 9 October 2007 - Revised: 26 February 2008 - Accepted: 3 March 2008 - Published: 13 May 2008
}

\begin{abstract}
Ion density enhancements at the topside lowlatitude ionosphere during a Bastille storm on 15-16 July 2000 and Halloween storms on 29-31 October 2003 were studied using data from ROCSAT-1/IPEI experiment. Prominent ion density enhancements demonstrate similar temporal dynamics both in the sunlit and in the nightside hemispheres. The ion density increases dramatically (up to two orders of magnitude) during the main phase of the geomagnetic storms and reaches peak values at the storm maximum. The density enhancements are mostly localized in the region of a South Atlantic Anomaly (SAA), which is characterized by very intense fluxes of energetic particles. The dynamics of nearEarth radiation was studied using SAMPEX/LEICA data on $>0.6 \mathrm{MeV}$ electrons and $>0.8 \mathrm{MeV}$ protons at around $600 \mathrm{~km}$ altitude. During the magnetic storms the energetic particle fluxes in the SAA region and in its vicinity increase more than three orders of magnitude. The location of increased fluxes overlaps well with the regions of ion density enhancements. Two mechanisms were considered to be responsible for the generation of storm-time ion density enhancements: prompt penetration of the interplanetary electric field and abundant ionization of the ionosphere by enhanced precipitation of energetic particles from the radiation belt.
\end{abstract}

Keywords. Ionosphere (Equatorial ionosphere; Ionospheric disturbances) - Magnetospheric physics (Geomagnetic storms; Energetic particles, precipitating; Magnetosphereionosphere interactions)

\section{Introduction}

Geomagnetic storms are accompanied with strong perturbations in the entire ionosphere, which have been observed using various techniques (e.g. Basu et al., 2001). Storm-time

Correspondence to: A. Dmitriev

(dalex@jupiter.ss.ncu.edu.tw) enhancements of ion and electron density (storm-enhanced density or SED) and temperature at the low- and mid-latitude topside ionosphere are detected by means of in-situ space experiments (e.g. Lin et al., 2005; Mannucci et al., 2005; Oyama et al., 2005; Yizengaw et al., 2005) and of remote techniques, such as ionosonde, radar and total electron content (TEC) obtained from GPS signal processing (Tsurutani et al., 2004; Yizengaw et al., 2005; Zhao, et al., 2005; Dabas et al., 2006; Becker-Guedes et al., 2007; Huang et al., 2007).

The observations show that the SED phenomenon is associated with an intensification of equatorial ionization anomaly (EIA), such that the F-layer peak height increases by a few hundred $\mathrm{km}$ and the EIA crest regions enlarge and move poleward by more than $10^{\circ}$. The dynamics is accompanied by strong variations of vertical (upward/downward) drift velocity with a magnitude of several hundred $\mathrm{m} / \mathrm{s}$. A prompt penetrating electric field, neutral wind disturbance dynamo electric field, and storm-time plasmapause dynamics are suggested to be responsible for such a pattern of ionospheric dynamics in the dayside hemisphere (e.g. Tsurutani et al., 2004, 2007; Unnikrishnan et al., 2005; Zhao et al., 2005). Storm-associated change in the thermospheric neutral wind pattern is considered to be a driving factor of disturbances in mid- and high-latitude ionosphere, especially in the nightside hemisphere (Ridley et al., 2003; Nicolls et al., 2006).

Storm-time prompt penetration of the interplanetary electric field to the mid- and low-latitude ionosphere is revealed experimentally (e.g. Kelley et al., 2003; Tsurutani et al., 2004; Lin and Yeh, 2005; Huang et al., 2007). There is practically no time delay for the penetration. The penetration efficiency is estimated at $\sim 10 \%$. Hence significant ionospheric effects require a very strong southward interplanetary magnetic field (IMF) and/or an extremely fast solar wind plasma flow.

Another source of the abundant ionization and heating at low- and mid-latitudes is the particle precipitation, which

Published by Copernicus Publications on behalf of the European Geosciences Union. 


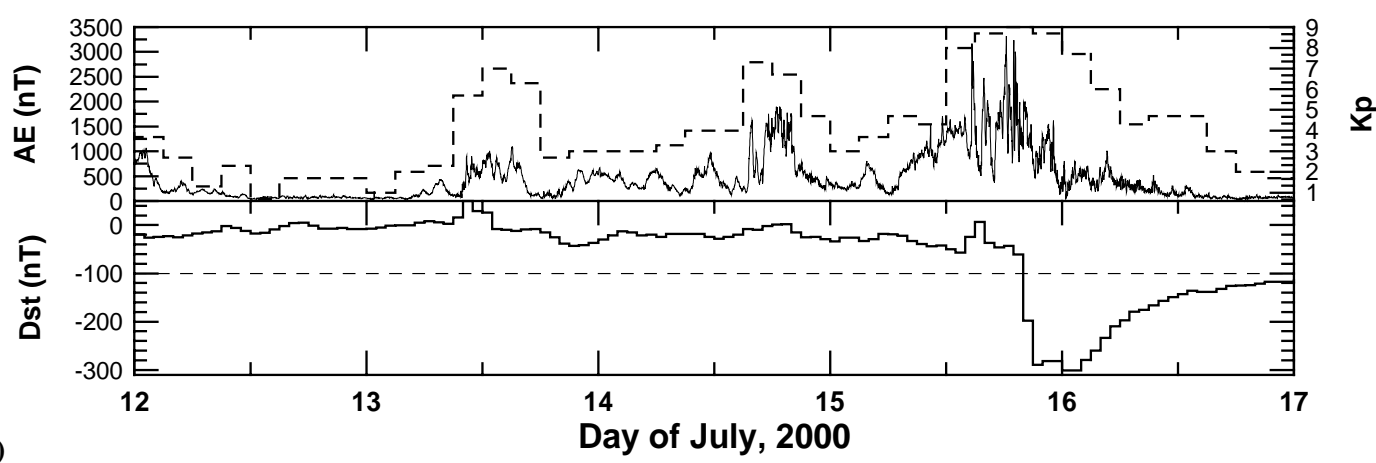

(a)

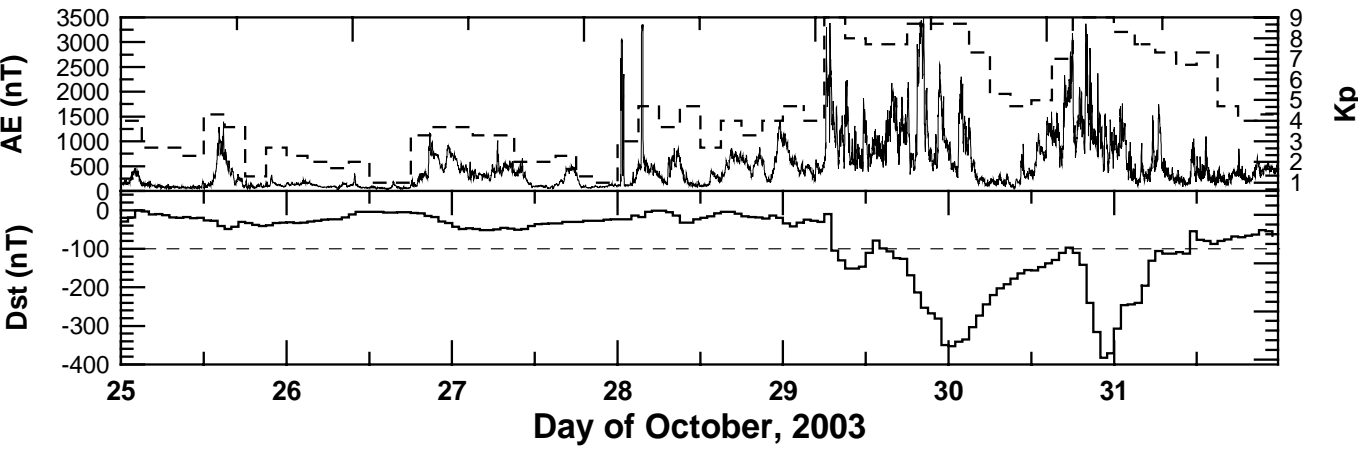

Fig. 1. Geomagnetic activity before and during (a) Bastille storm and (b) Halloween storms. Auroral AE index (solid curve, left axis) and mid-latitude $K_{p}$ index (dashed histogram, right axis) are presented at upper panels. Storm-time $D_{s t}$-variation is shown at lower panels.

is intensified during magnetic storms. Fluxes of energetic neutral atoms penetrating to the ionosphere are generated through charge-exchange interactions of the ring current ions with the plasmaspheric neutrals (Tinsley, 1979; Tinsley et al., 1988; Kozyra and Nagy, 1991). In the maximum phase of great magnetic storms, the precipitation of energetic protons, which populate the field-aligned currents, can move to middle latitudes of $\sim 45^{\circ}$ (Wang et al., 2006).

Based on analyses of the Bastille magnetic storm on 1617 July, Lin and Yeh (2005) proposed that energetic particle precipitation plays an important role in the SEDs observed in the South Atlantic Anomaly (SAA) region on the nightside. They demonstrate that the precipitation of energetic electrons from the radiation belt in the SAA region significantly increases Hall conductivity in the E-region. Inside and above that high-conductance region, the electric fields are reduced and the ion density enhances. However, the regions with low conductivity are characterized by enhanced electric field and depleted ion density.

Precipitation of energetic particles from the Earth radiation belts (ERB) is a general feature of geomagnetic storms (e.g. Rodger et al., 2006; Millan and Thorne, 2007). The middle and high latitudes are affected by intense precipitation of energetic electrons, which is also considered as a powerful source of ionization in the upper atmosphere and ionosphere (Baker et al., 1987). Very intense precipitation of the energetic electrons occurred during geomagnetic storms at the southern edge of SAA in the slot region of the electron radiation belt (Dmitriev et al., 2000). During the Halloween magnetic storms in October-November the precipitation was so intensive that the belt of energetic protons almost completely disappeared (Looper et al., 2005).

In the present study we analyze enhancements of ion density and precipitation radiation during Bastille and Halloween geomagnetic storms. In the first part, topside ionospheric disturbances are investigated using experimental data from the ROCSAT-1 satellite. SAMPEX observations of the near-Earth fluxes of energetic particles are compared with the ionospheric disturbances in the second part. In the third part we discuss the possible physical mechanisms of the relationship between the enhancements of ionospheric ionization and precipitation of the energetic particles. The last part presents the conclusion.

\section{Storm-time ionospheric disturbances}

Geomagnetic conditions before and during the Bastille and Halloween storms are shown in Fig. 1. There were no strong geomagnetic storms with $D_{s t}<-100 \mathrm{nT}$ within several days preceding the great storms on 15 July 2000 and 29 October 2003. The dynamics of the $\mathrm{AE}$ and $K_{p}$ indices before the storms show an enhanced auroral activity, which is, however, much weaker than those of the storm-time disturbances. 


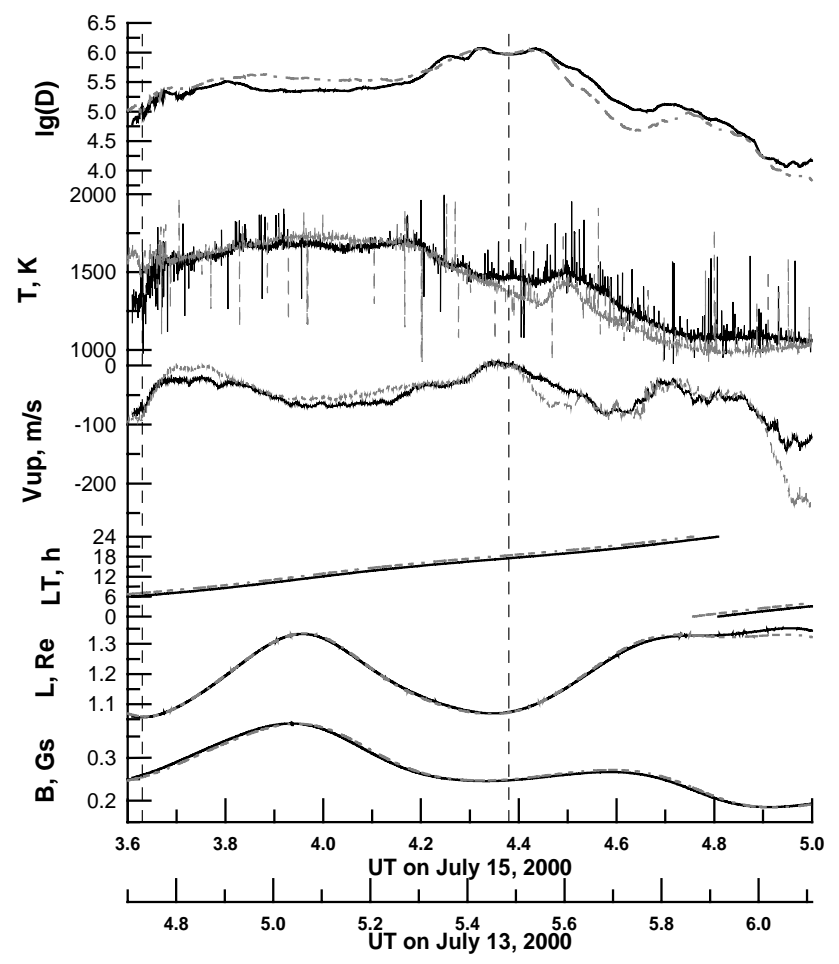

Fig. 2. Comparison of prestorm ionospheric conditions on 15 July (black solid line) with quiet day on 13 July (gray dashed lines) for overlapping orbits of the ROCSAT-1 satellite. Passing the ionospheric regions with practically the same local time LT, magnetic field $\boldsymbol{B}$ and drift shells $L$, the satellite observes very close time profiles of the ion density $D$, temperature $T$ and upward velocity $V_{\text {up }}$. Vertical dashed lines depict the geomagnetic equator.

Hence in a first approach we can consider the time intervals on 12-14 July 2000 and 25-28 October 2003 as quiet or relatively weak disturbed days.

Our study of storm-time conditions in the topside ionosphere is based on experimental data from the near-Earth ROCSAT-1 satellite which has a circular orbit with $35^{\circ}$ inclination and is at an $\sim 600 \mathrm{~km}$ altitude (Chang et al., 1999). The ionospheric Plasma and Electrodynamics Instrument (IPEI) on board ROCSAT-1 provides experimental information about the characteristic features of ionospheric ion density, temperature, composition and drift velocity (Yeh et al., 1999). In the present study we use 1-s resolution IPEI data of the ion density, temperature and vertical drift velocity for analyses.

The ROCSAT-1 orbital characteristics provide us an opportunity to find overlapping orbits within four successive days. The overlapping orbits have a very close location, i.e. the satellite passes the same geographical regions at practically the same local time. An example of the ROCSAT1/IPEI observations during such overlapping orbits on 13 July and 15 July 2000 is presented in Fig. 2. The time profiles of ionospheric parameters are pretty close for the over-

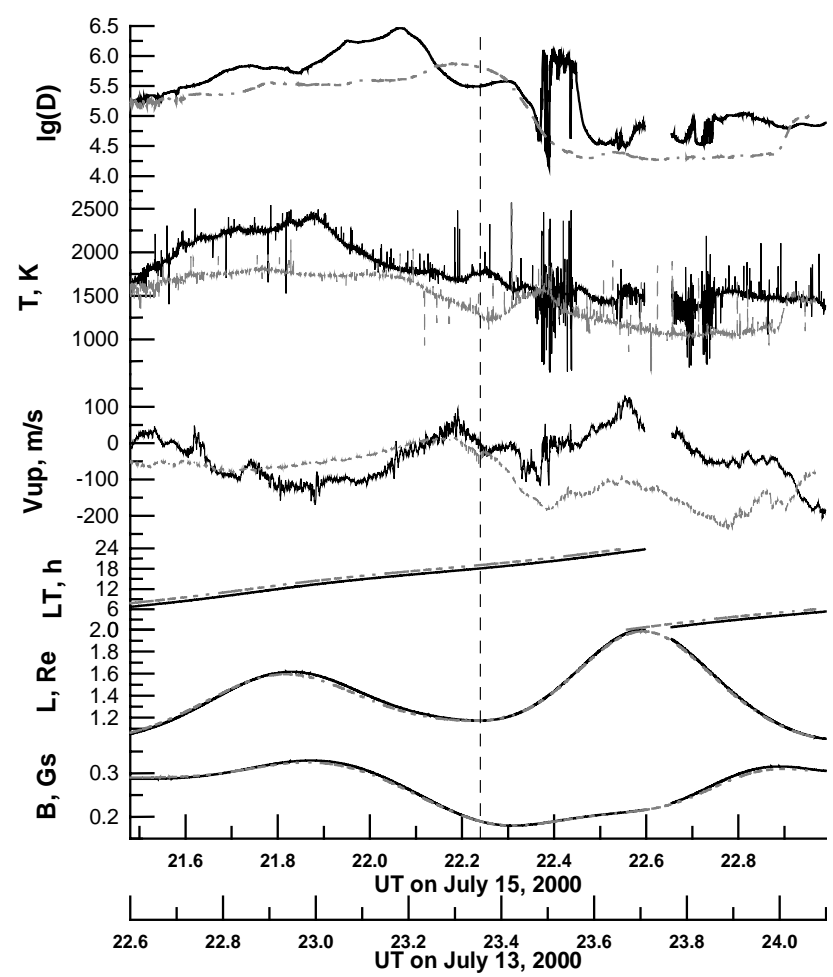

Fig. 3. Comparison of storm-time ionospheric disturbances on 15 July (black solid line) with quiet conditions on 13 July (gray dashed lines) for overlapping orbits of the ROCSAT-1 satellite (from top to bottom): ion density in logarithmic scale, ion temperature and upward velocity, local time, L-shell, and magnetic field strength. Vertical dashed line depicts the geomagnetic equator. The disturbed ionospheric conditions are substantially different from the quiet conditions.

lapping orbits during the quiet and the slightly disturbed intervals. The observed differences in ion density and temperature under the two geomagnetic activity conditions are not very large, except for the dawn sector ( 06:00 LT). They are caused partially by the geomagnetic activity, which enhances slightly by 15 July. Large differences observed near 06:00 LT are probably due to slightly different local times associated with overlapping passes. In the dawn sector the ionospheric conditions change rapidly with local time, in particular, during the sunrise. Hence in that local time sector a small difference in the LT leads to a significant difference in the measurements.

In Fig. 3 we compare storm-time ionospheric disturbances during the main phase of the Bastille storm (21:30 23:00 UT) on 15 July with those during the quiet conditions on 13 July 2000. During that time interval ROCSAT1 crosses the geomagnetic equator at the local morning and evening, such that its passes of the dayside and nightside ionosphere are situated, respectively, in the Northern and Southern Hemispheres. During the geomagnetic storm the ROCSAT-1/IPEI observes significant growth of the ion 


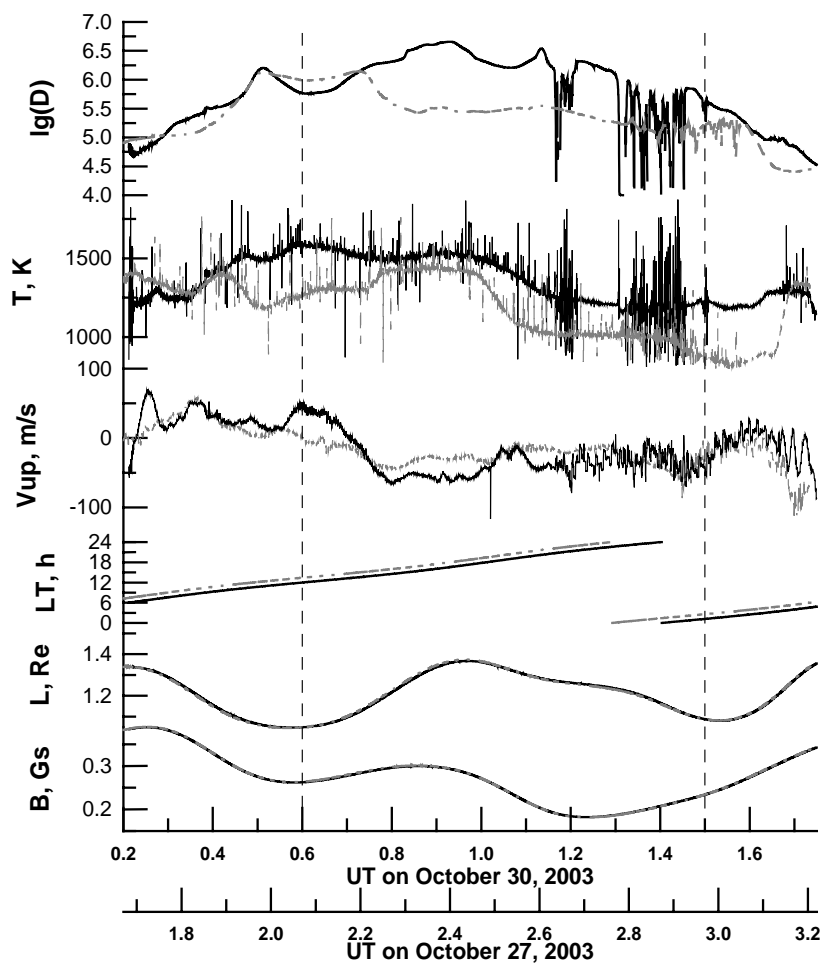

Fig. 4. The same as in Fig. 3 but for storm-time disturbances on 30 October 2003 and quiet conditions on 27 October 2003.

density and temperature in both the dayside and the nightside ionosphere. Most prominent increases of ion density are observed in the crest regions, which are extended poleward. At the same time the ion density at the geomagnetic equator is depleted. It is interesting to note a nightside feature observed during 22:22-22:27 UT ( 20:20 LT) in the Southern Hemisphere, which is characterized by extremely high variations of ion density with an amplitude of $\sim 1.5$ order of magnitude above the background value. That feature is accompanied by very strong variations in the temperature and vertical plasma drift velocity of $\sim 100 \mathrm{~m} / \mathrm{s}$. A possible nature of such ionospheric disturbances is discussed by Lin and Yeh (2005).

Topside ionospheric disturbances at the maximum of the great Halloween geomagnetic storm (during 00:1001:40 UT) on 30 October 2003 are presented in Fig. 4. During that time the ascending and descending parts of the ROCSAT- 1 orbit are located, respectively, in the nightside and dayside hemispheres, such that the satellite crosses the geomagnetic equator in close vicinity of local midnight and noon. Most prominent ion density enhancement observed at 00:45-01:20 UT in the Southern Hemisphere has a two-peak structure. The first maximum at $\sim 00: 55 \mathrm{UT}(\sim 16: 20 \mathrm{LT})$ and the second one at $\sim 01: 09 \mathrm{UT}(\sim 20: 30 \mathrm{LT})$ appeared, respectively, before and after the sunset. Both of them are characterized by an enhanced temperature and a relatively slow downward plasma drift of about $50 \mathrm{~m} / \mathrm{s}$. Similar to that of the Bastille storm, the ion density is significantly depleted at the geomagnetic equator.

The first maximum can be attributed to the crest region, which is extended far toward the South Pole. However, it is rather difficult to explain the origin of the second maximum, whose location at middle latitudes is pretty far from the geomagnetic equator. It is important to note that despite that second maximum is situated at nightside, the ion density in that region is comparable with the dayside maximum. The former one even exceeds the dayside crest in the Northern Hemisphere, which indicates a substantial north-south asymmetry of the topside ionosphere.

In order to study the effect of storm-enhanced density (SED) numerically, we introduce a ratio of the ion densities:
$r D=\left(D_{d}-D_{0}\right) / D_{0}$
if
$D_{d} \geq D_{0}$
$r D=\left(D_{d}-D_{0}\right) / D_{d}$
if
$D_{d}<D_{0}$,

where $D_{0}$ and $D_{d}$ are the ion densities measured with 1-s resolution during the quiet and storm time, respectively. In this way we obtain the profiles of ion density relative variations during the Bastille and Halloween storms. Using these profiles we can determine the maximum $r$ Dmax of the ratio $r D$ for each of the dayside and nightside passes. So the time step of the rDmax is $\sim 50 \mathrm{~min}$, which is about half of the orbital period.

Storm-time variations of the rDmax are presented in Fig. 5 . One can clearly see that during the main phase of geomagnetic storms the ion density increases significantly on both sunlit and nightside hemispheres. The most intense SEDs are observed in the dusk sector. At dayside the rDmax reaches peak values with an amplitude of $>10$ at the maximum of storms and then the rDmax decreases within several hours. The rDmax peaks are located in the afternoon sector, while the decreases of rDmax are found in the noon and prenoon sectors.

The dynamics of ion density at nightside is more complicated such that the peaks of rDmax can occur during the recovery phase at various local times: from the dusk to postmidnight sector. In Fig. 5 we can see that the nightside SEDs can be accompanied with both northward and southward IMF orientation. The peaks and/or enhancements of $r D \max$ in the evening of 15 July 2000, in the evenings of 29 and 30 October 2003, are observed during long-lasting intervals of very strong southward IMF. On the other hand, the peaks of $r D$ max can be found in the morning on 16 July 2000, at noon on 29 October 2003 and on 31 October 2003, when the longlasting northward IMF dominates and geomagnetic disturbances at that time are caused by perturbations in the solar wind plasma rather than by the IMF orientation.

The observed variations of $r D \max$ are contributed by temporal and spatial variations of the ion density, which is detected by the satellite passing different spatial regions at a different time. Spatial location of most prominent SEDs with 


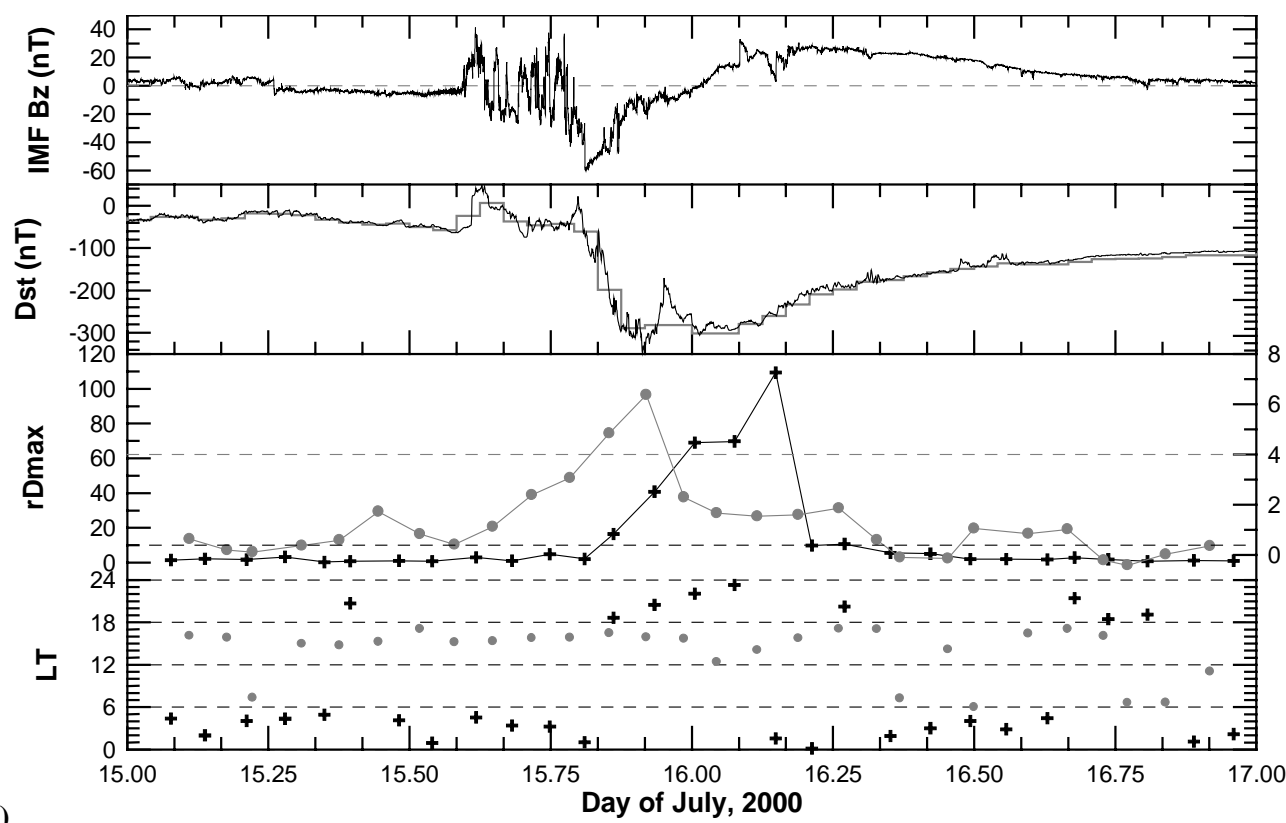

(a)

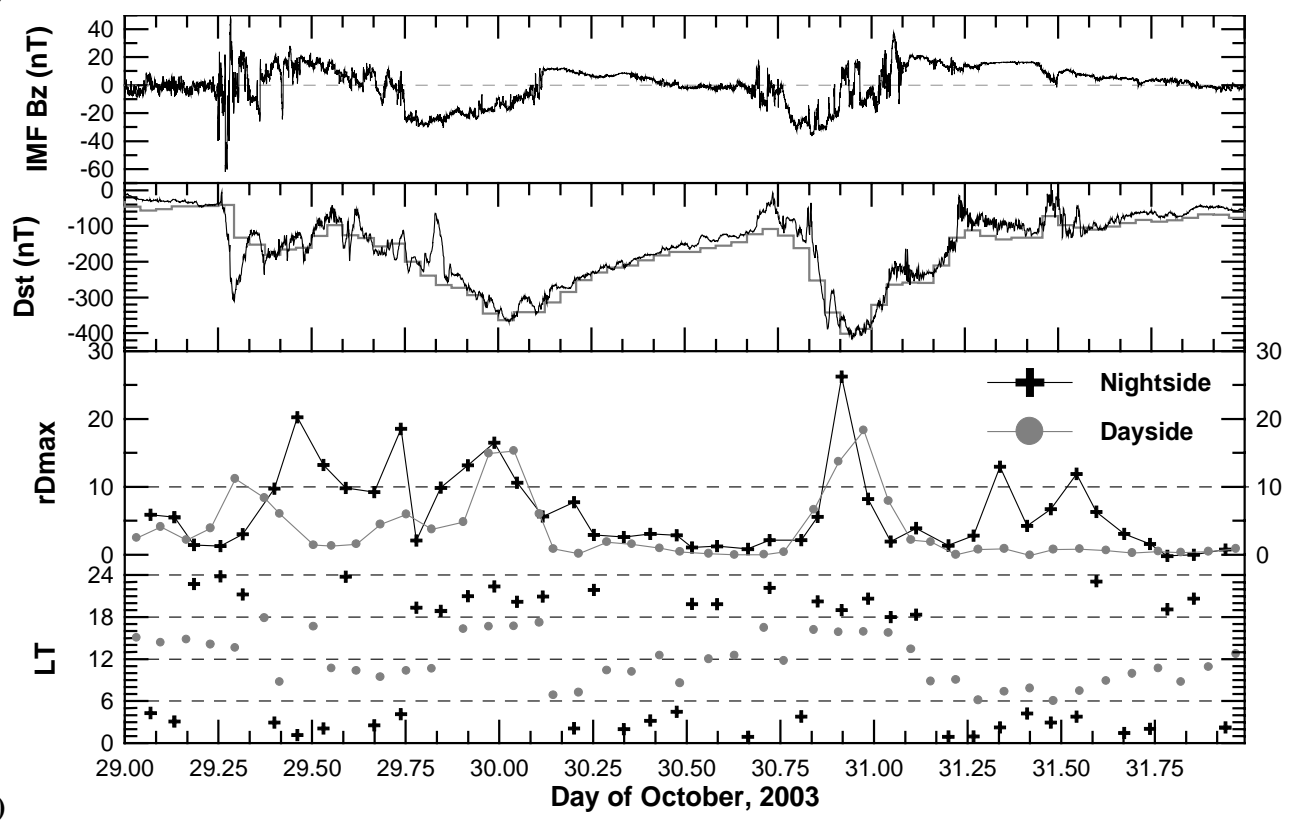

Fig. 5. Ion density enhancements during (a) Bastille storm on 15-16 July 2000 and (b) Halloween storms on 29-31 October 2003. The panels show from top to bottom: large variations of the IMF $B z$ measured by the ACE satellite; storm-time 1-h and 1-min $D_{s t}$-variation (gray histogram and black curve, respectively); maximal ion density enhancements $r$ Dmax detected at the low-latitude topside ionosphere by the ROCSAT-1/IPEI during dayside (gray circles, right axis) and nightside (black crosses, left axis) passes; and local time of the corresponding enhancements. Most prominent ion density enhancements both in sunlit and nightside ionosphere group around the maximum of magnetic storms.

$r D>10$ is presented in Fig. 6 in a geographic coordinate system. Note that during the Bastille storm (Fig. 6a) the threshold for prominent enhancements was accepted to be $r D=4$ in the dayside ionosphere, which corresponds to the ROCSAT1 passes in the Northern Hemisphere. As seen in Fig. 6, the enhancements cluster at longitudes from $200^{\circ}$ to $360^{\circ}$ and latitudes from $-10^{\circ}$ to $-35^{\circ}$ in the Southern Hemisphere. That region is overlapped with the location of SAA. Note that during the Bastille storm ROCSAT-1 passes the SAA region at nighttime only, while during the Halloween event the SAA is passed during both daytime (descending orbits) and nighttime (ascending orbits). 


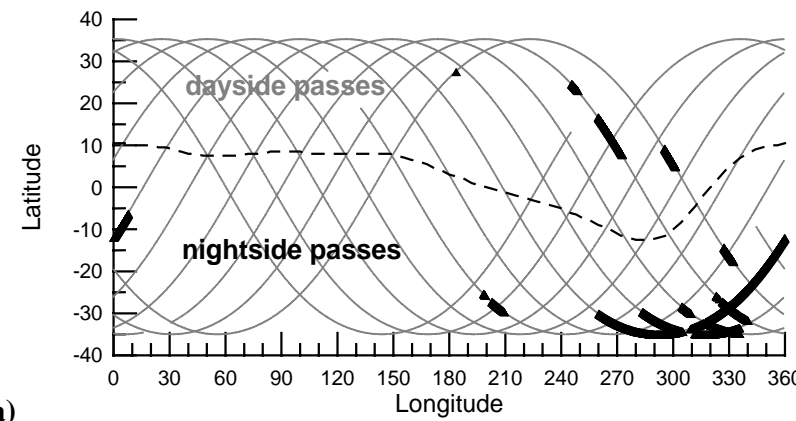

(a)

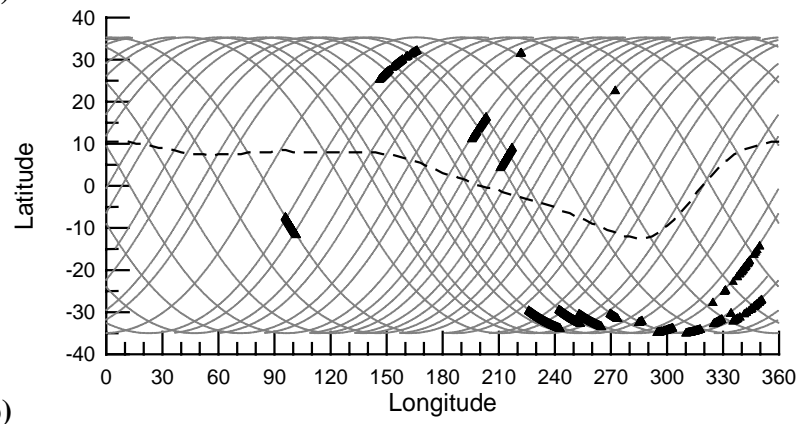

Fig. 6. Geographical location of most prominent ion density enhancements (black triangles) observed by the ROCSAT-1/IPEI during (a) Bastille and (b) Halloween storms. The ROCSAT orbits are shown by gray dotted lines. The thick dashed line indicates the geomagnetic equator. The vast majority of the enhancements with $r D>10$ is located in the South Atlantic region. During the Halloween event the ascending and descending parts of the ROCSAT-1 orbit correspond, respectively, to the nighttime and daytime passes.

The SEDs in the Northern Hemisphere might be associated with the enlarged crest regions. For some of them we can find corresponding enhancements in the magnetically conjugated regions. However most of prominent enhancements in the Southern Hemisphere have no conjugated enhancements in the Northern Hemisphere, despite that ROCSAT-1 passes the corresponding regions. Note that such significant northsouth asymmetry of the topside ionosphere is observed in the summertime during the Bastille storm and near equinox during the Halloween storms. Hence the asymmetry can not be a result of seasonal effect. It should be caused by another natural reason.

\section{Energetic particle precipitation}

Energetic particle fluxes at altitudes of the ROCSAT- 1 orbit are studied using experimental data of electrons with energy $>0.6 \mathrm{MeV}$ and protons with energy $>0.8 \mathrm{MeV}$ measured by Low Energy Ion Composition Analyzer (LEICA) on board the SAMPEX satellite (Baker et al., 1993; Mason et al., 1993). The SAMPEX satellite has a slightly elliptical polar orbit at heights of $500 \mathrm{~km}$ to $600 \mathrm{~km}$.

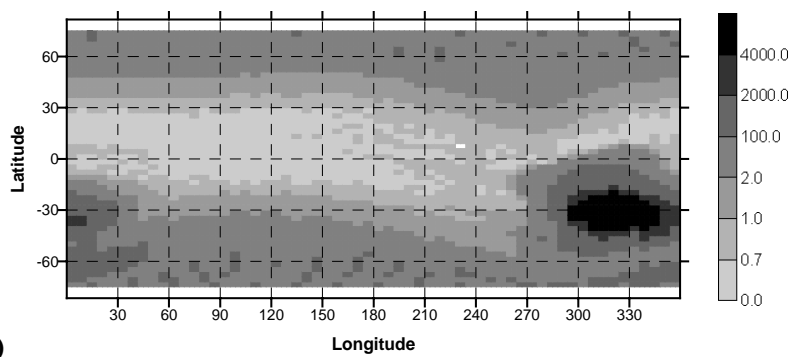

(a)

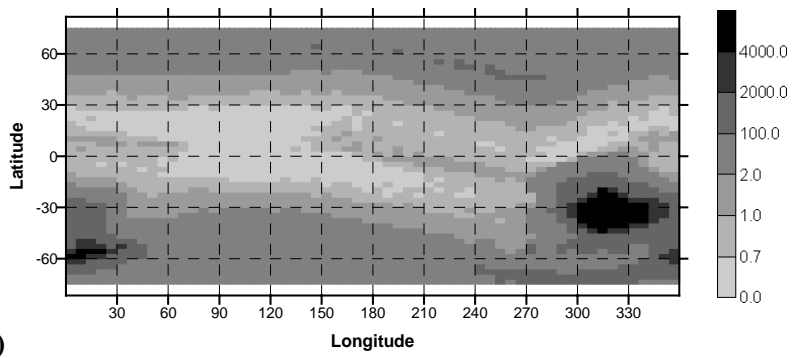

Fig. 7. Geographical distribution of quiet-time fluxes of $>0.6$ electrons and $>0.8$ protons measured by the SAMPEX/LEICA on July 2000 (a) and October 2003 (b). The intensities are indicated in a gray scale from $<0.7 \mathrm{~cm}^{-2} \mathrm{~s}^{-1} \mathrm{sr}^{-1}$ (light gray) to $>4000 \mathrm{~cm}^{-2} \mathrm{~s}^{-1} \mathrm{sr}^{-1}$ (black). Most intense fluxes at middle latitudes $\left(<40^{\circ}\right)$ are observed in the region of South Atlantic Anomaly.

Figure 7 shows a geographical distribution of energetic particle fluxes observed by the SAMPEX/LEICA during quiet days preceding the Bastille and Halloween storms. The distribution is constructed on the base of a grid with $3^{\circ}$ and $6^{\circ}$ step, respectively, in latitude and longitude. The average of the particle fluxes is calculated for each cell of the grid.

The quiet-day fluxes vary from very weak values of $<0.7 \mathrm{~cm}^{-2} \mathrm{~s}^{-1} \mathrm{sr}^{-1}$ at the equator to very high intensities of $>4000 \mathrm{~cm}^{-2} \mathrm{~s}^{-1} \mathrm{sr}^{-1}$ (up to $10^{5} \mathrm{~cm}^{-2} \mathrm{~s}^{-1} \mathrm{sr}^{-1}$ ) in the SAA region. The latter is situated in the range of latitudes from $-10^{\circ}$ to $-45^{\circ}$ and longitudes from $290^{\circ}$ to $360^{\circ}$. It is interesting to note a solar cycle variation of the energetic particle fluxes in the SAA region: during the solar maximum on July 2000 , high intensities (say $>2000 \mathrm{~cm}^{-2} \mathrm{~s}^{-1} \mathrm{sr}^{-1}$ ) occupy a larger area than that on October 2003, i.e. on the declining phase of solar cycle.

Figure 8 shows energetic particle fluxes detected by the SAMPEX/LEICA during the main phase, maximum and beginning of the recovery phase of the geomagnetic storms. Namely, according to Fig. 5, the disturbed intervals occur from 18:00 UT on 15 July to 06:00 UT on 16 July 2000 for the Bastille storm, from 06:00 UT to 14:00 UT on 29 October 2003, from 18:00 UT on 29 October to 03:00 UT on 30 October 2003 and from 19:00 UT on 30 October to 02:00 UT on 31 October 2003 for the Halloween storms.

We can distinguish between several different regions of intense particle fluxes. At middle and high latitudes one can see intense sporadic enhancements, which are caused by two 


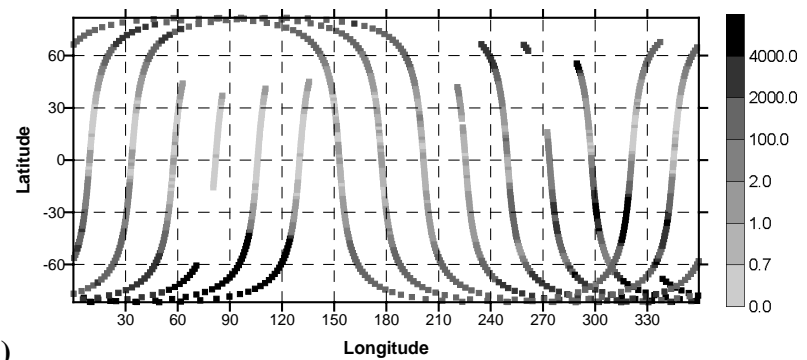

(a)
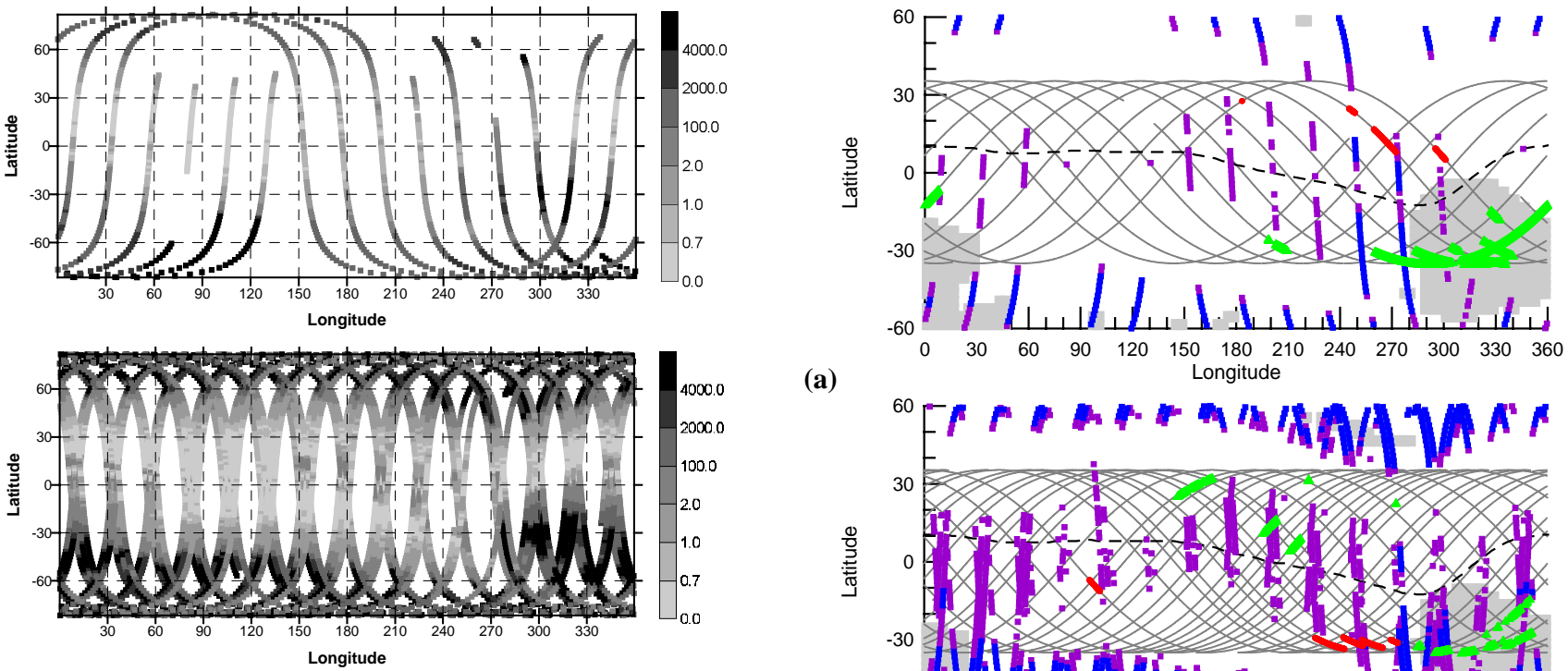

(b)

Longitude

(a)

Fig. 8. Fluxes of $>0.6$ electrons and $>0.8$ protons measured by the SAMPEX/LEICA during the great geomagnetic storms on 1516 July 2000 (a) and 29-31 October 2003 (b). The intensities are indicated in a gray scale from $<0.7 \mathrm{~cm}^{-2} \mathrm{~s}^{-1} \mathrm{sr}^{-1}$ (light gray) up to $10^{5} \mathrm{~cm}^{-2} \mathrm{~s}^{-1} \mathrm{sr}^{-1}$ (black).

concurrent effects: direct penetration of solar energetic particles (SEP) to the polar caps and storm-time particle precipitation from the outer radiation belt (e.g. Panasyuk et al., 2004; Malandraki et al., 2005). Most intense fluxes are observed in the SAA and in the slot region between the SAA and outer radiation belt at latitudes of $-60^{\circ}$ to $-40^{\circ}$. Those fluxes are associated with very intense precipitation of energetic particles from the Earth's radiation belt during great geomagnetic storms (Dmitriev et al., 2000; Panasyuk et al., 2004; Looper et al., 2005). It is interesting to note an intensification of particle fluxes at low latitudes in the longitudinal range from $150^{\circ}$ to $360^{\circ}$.

For numerical estimation of the energetic particle enhancements we introduce a ratio

$r I=I_{d} / I_{o}$,

where $I_{d}$ is a flux of $>0.6 \mathrm{MeV}$ electrons and $>0.8 \mathrm{MeV}$ protons during the disturbed periods of geomagnetic storms and $I_{0}$ is the quiet-time average flux in the cell of a corresponding location (see Fig. 7). The ratio $r I$ is calculated along the SAMPEX orbit during the disturbed times of Bastille and Halloween storms. In Fig. 9 we show the regions of enhanced ratio $r I$ during the disturbed intervals. At that time the fluxes of energetic particles increase up to four orders of magnitude, i.e. $r I=10^{4}$.

Most prominent particle enhancements are associated with the precipitation from the outer radiation belt. During strong magnetic storms the regions of particle precipitation come down to lower latitudes, such that very strong particle en-

(b)

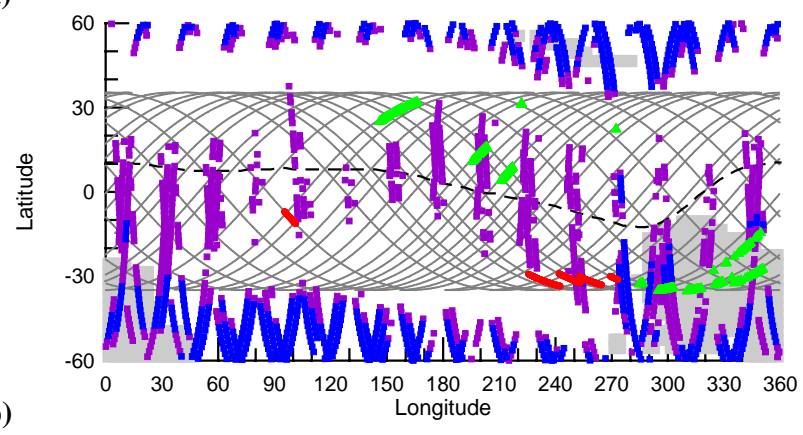

Fig. 9. Significant enhancements of the ion density observed by the ROCSAT-1/IPEI in the dayside (red circles) and in the nightside (green triangles) hemispheres, and energetic particle enhancements (squares) observed by the SAMPEX/LEICA at altitudes $\sim 600 \mathrm{~km}$ during the Bastille (a) and Halloween (b) geomagnetic storms. The orbit of ROCSAT-1 during those disturbed periods is shown by gray dotted line. The relative enhancements of particle fluxes of $r I>1.3$ and $r I>10$ are indicated, respectively, by violet and blue squares. For reference, gray areas depict the regions of strong background particle fluxes (including SAA) with quiet-time intensities of $>100 \mathrm{~cm}^{-2} \mathrm{~s}^{-1} \mathrm{sr}^{-1}$. Thick dashed line indicates the geomagnetic equator. Most of the ionospheric ion density enhancements are overlapped with the regions of enhanced energetic particle fluxes.

hancements are observed at latitudes of $\sim 40^{\circ}$ above Australia and North America. That is very close but still beyond the ROCSAT- 1 orbital range of $35^{\circ}$ latitude. As a result, we do not find substantial ion density enhancements in those particular regions.

Prominent particle enhancements are also observed inside and around the SAA region. In Fig. 9 one can clearly see that at the latitudes of the ROCSAT-1 orbit significant SEDs with $r D>10$ are well overlapped with that region. We should note that those enhancements are also overlapped in time, because they are observed within the same disturbed time intervals. Moreover, that effect persists both in the nightside and in the dayside hemispheres, which corresponds, respectively, to the ascending and the descending parts of the ROCSAT- 1 orbit during the Halloween storms. The observed overlapping of the SEDs and energetic particle enhancements hence reveals their physical relationship. 


\section{Discussion}

ROCSAT-1/IPEI observations of the storm-time ion density enhancements in the dayside ionosphere support well the mechanism of prompt penetration of the interplanetary electric field (Tsurutani et al., 2004, 2007). The SEDs are observed during the main phase of the Bastille and Halloween geomagnetic storms, when the strong IMF is directed southward (see Fig. 5). The spatial structure of the EIA clearly resembles a "daytime super-fountain" effect (Manucci et al., 2005). Namely, the density is depleted and enhanced, respectively, at the geomagnetic equator and in the crest regions, which are enlarged and shifted to higher latitudes (see Figs. 3 and 4).

The observed nightside dynamics of the ion density is more complicated. In Fig. 5 we found that the temporal patterns of SEDs in the nightside and dayside hemispheres are very close. Namely, both in the dayside and nightside ionosphere, the ion densities increase practically simultaneously during the main phase and reach peak values at the maximum of magnetic storms. Such a dynamic pattern of the nightside SEDs can not be explained by only the effect of prompt electric field penetration. Tsurutani et al. (2004) show that the dawn to dusk electric field penetrating in the nightside hemisphere during the main phase of magnetic storm, push the ionospheric plasma downward, leading to a decrease in the ionospheric TEC by recombination. Due to the negative gradient of ionization in the topside ionosphere, the downward plasma drift should also lead to a decrease in the ion density at a given altitude. On the other hand, the appearance of nighttime SEDs during long-lasting northward IMF might be explained by a penetration of the strong dusk to dawn electric field. However, possibility and conditions for that penetration to the "closed" magnetosphere seems not so obvious.

We have found that during the Bastille and Halloween storms the location of strong SEDs (more than one order of magnitude) overlaps well with the regions of intense precipitation of the energetic particles on both the sunlit and nighttime ionosphere (see Fig. 9). The vast majority of the most pronounced ion density enhancements groups around the SAA region, where the storm-time fluxes of energetic particles increase by 1 to 3 orders of magnitude.

Analysis of the ion temperature and drift velocity in the SAA region shows that the ion enhancements are accompanied by the enhanced temperature, but the vertical ion drift can be relatively small. Hence the regions of ion density enhancements are characterized by plasma heating without significant vertical bulk motion that might indicate an effect of direct ionization.

Very intense fluxes of precipitating energetic particles can provide significant ionization of the ionosphere and upper atmosphere. Numerical estimation of their contribution to the topside ionosphere requires additional information about the spectral characteristics of proton and electron fluxes and pitch-angle distributions of the particles. Baker et al. (1987) estimate roughly that precipitation of $>1 \mathrm{MeV}$ electrons contributes the energy comparable to $\sim 0.1 \%$ of a major geomagnetic storm's total energy dissipation. But that energy deposition would be in a very specialized location compared to the ionospheric Joule heating and ring current formation that dominates in a typical storm.

More difficulty arises due to the sporadic character of the particle precipitation. It is rather difficult to find both spatial and temporal coincidence between the SEDs observed by one satellite and intensification of the energetic particle fluxes observed by another satellite. Complex observations of ionospheric plasma and energetic particles, especially electrons, on board one satellite could be very useful for a quantitative study of the magnetosphere-ionosphere coupling phenomena.

Strong topside ion density and TEC enhancements in the American sector during several strong geomagnetic storms, including the Bastille and Halloween events, are also reported by Foster and Coster (2007). The enhancements are observed during the main phase in the dusk sector and corotate into the nightside hemisphere. The SED effect is accompanied by a strong uplift of the equatorial F-layer up to altitudes of $\sim 600 \mathrm{~km}$. The authors suggest that the dominant mechanism for producing that effect is the plasma transport associated with disturbance electric fields, which are generated in the SAA region due to the presence of conductivity gradients. During the geomagnetic storms the increased conductivity gradients are produced by the particle precipitation in the SAA region. A combination of the penetrated interplanetary electric field and local electric fields generated in the SAA region at dusk is considered as a driver of the Flayer uplifting.

During the Halloween storms the most prominent ion density enhancements are observed by the ROCSAT-1/IPEI in the SAA region at $\sim 16: 00 \mathrm{LT}$, i.e. in the dusk sector. However, during the Bastille storm the largest SED effect with $r D>100$ is observed in the SAA region at $\sim 02: 00 \mathrm{LT}$, i.e. in the postmidnight sector. To explain the SED effect at nighttime topside ionosphere Lin and Yeh (2005) have proposed a physical mechanism of electric field generation due to a change in conductivity in the E-layer during the intense particle precipitation in the SAA region.

\section{Conclusion}

Very strong ion density enhancements up to two orders of magnitude have been observed by the ROCSAT-1/IPEI in the topside ionosphere at low latitudes during the Bastille and Halloween magnetic storms. The enhancements occur in a wide range of longitudes both in the dayside and nightside hemispheres. We can suggest two main mechanisms, which are responsible for the generation of the observed SEDs. The first one is the prompt penetration of the interplanetary 
electric field, which causes an expansion of the EIA crest regions toward the higher latitudes during the main phase of geomagnetic storms. The second mechanism is associated with abundant ionization of the ionosphere by strongly enhanced precipitation of energetic particles from the radiation belt during geomagnetic storms. The SAMPEX/LEICA observations of the energetic particles show that the most intense precipitation at middle and low latitudes is localized in the South Atlantic Anomaly region, where most of the SEDs are observed by the ROCSAT-1/IPEI. In addition to a direct ionization effect, the enhanced particle precipitation in the SAA region causes an increase in the ionospheric conductivity, growth of the conductivity gradients and, as a result, generation of strong local electric fields, which are able to produce strong upward/dawnward drifts of the highly ionized plasma.

Acknowledgements. The authors thank G. Maeon at LHU/APL and CDAWeb for providing data from the SAMPEX satellite, NASA/GSFC ISTP for providing data from the ACE satellite and Kyoto World Data Center for Geomagnetism for providing the $D_{s t}$, ASY/SYM and AE geomagnetic indices. This work was supported by grants NSC-94-2811-M-008-039, NSC-95-2811-M-008020 and NSC96-2923-M-008-001MY3/07-02-92004HHC_a from the National Science Council of Taiwan.

Topical Editor M. Pinnock thanks O. Sheyner and another anonymous referee for their help in evaluating this paper.

\section{References}

Baker, D. N., Blake, J. B., Gorney, D. J., Higbie, P. R., Klebesadel, R. W., and King, J. H.: Highly relativistic magnetospheric electrons: A role in coupling to the middle atmosphere, Geophys. Res. Lett., 14, 1027-1030, 1987.

Baker, D. N., Mason, G. M., Figueroa, O., Colon, G., Watzin, J. G., and Aleman, R. M.: An Overview of the Solar, Anomalous, and Magnetospheric Particle Explorer (SAMPEX) Mission, IEEE T. Geosci. Remote, 31, 531-541, 1993.

Basu, S., Basu, S., Valladares, C. E., Yeh, H.-C., Su, S.-Y., MacKenzie, E., Sultan, P. J., et al.: Ionospheric effects of major magnetic storms during the International Space Weather Period of September and October 1999: GPS observations, VHF/UHF scintillations, and in situ density structures at middle and equatorial latitudes, J. Geophys. Res., 106(A12), 30 389-30 413, 2001.

Becker-Guedes, F., Sahai, Y., Fagundes, P. R., Espinoza, E. S., Pillat, V. G., Lima, W. L. C., Basu, Su., et al.: The ionospheric response in the Brazilian sector during the super geomagnetic storm on 20 November 2003, Ann. Geophys., 25, 863-873, 2007, http://www.ann-geophys.net/25/863/2007/.

Chang, Y.-S., Chiang, W.-L., Yiang, S.-J., Holt, B. J., Lippincott, C. R., and Hsieh, K. C.: System Architecture of the IPEI Payload Onboard ROCSAT-1, Terr. Atmos. Ocean Sci., Supplementary Issue, 7-18, March 1999.

Dabas, R. S., Das, R. M., Vohra, V. K., and Devasia, C. V.: Space weather impact on the equatorial and low latitude F-region ionosphere over India, Ann. Geophys., 24, 97-105, 2006, http://www.ann-geophys.net/24/97/2006/.
Dmitriev, A. V., Minaeva, Yu. S., and Orlov, Yu. V.: Model of the slot region of Earth's electron radiation belt depending on the heliospheric parameters, Adv. Space Res., 25, 2311-2314, 2000.

Foster, J. C. and Coster, A. J.: Conjugate localized enhancement of total electron content at low latitudes in the American sector, J. Atmos. Sol.-Terr. Phy., 69, 1241-1252, 2007.

Huang, C.-S., Sazykin, S., Chau, J. L., Maruyama, N., and Kelley, M. C.: Penetration electric fields: Efficiency and characteristic time scale, J. Atmos. Sol.-Terr. Phy., 69, 1135-1146, 2007.

Kelley, M. C., Makela, J. J., Chau, J. L., and Nicolls, M. J.: Penetration of the solar wind electric field into the magnetosphere/ionosphere system, Geophys. Res. Lett., 30(4), 1158, doi:10.1029/2002GL016321, 2003.

Kozyra, J. U. and Nagy, A. F.: Ring current decay - coupling of ring current energy into the thermosphere/ionosphere system, J. Geomagn. Geoelectr., 43, Suppl., 285-297, 1991.

Lin, C. S. and Yeh, H. C.: Satellite observations of electric fields in the South Atlantic anomaly region during the July 2000 magnetic storm, J. Geophys. Res., 110, A03305, doi:10.1029/2003JA010215, 2005.

Lin, C. H., Richmond, A. D., Liu, J. Y., Yeh, H. C., Paxton, L. J., Lu, G., Tsai, H. F., and Su, S.-Y.: Large-scale variations of the low-latitude ionosphere during the October-November 2003 superstorm: Observational results, J. Geophys. Res., 110, A09S28, doi:10.1029/2004JA010900, 2005.

Looper, M. D., Blake, J. B., and Mewaldt, R. A.: Response of the inner radiation belt to the violent Sun-Earth connection events of October-November 2003, Geophys. Res. Lett., 32, L03S06, doi:10.1029/2004GL021502, 2005.

Malandraki, O. E., Lario, D., Lanzerotti, L. J., Sarris, E. T., Geranios, A., and Tsiropoula, G.: October/November 2003 interplanetary coronal mass ejections: ACE/EPAM solar energetic particle observations, J. Geophys. Res., 110, A09S06, doi:10.1029/2004JA010926, 2005.

Mannucci, A. J., Tsurutani, B. T., Iijima, B. A., Komjathy, A., Saito, A., Gonzalez, W. D., Guarnieri, F. L., Kozyra, J. U., and Skoug, R.: Dayside global ionospheric response to the major interplanetary events of October 29-30, 2003 "Halloween Storms", Geophys. Res. Lett., 32, L12S02, doi:10.1029/2004GL021467, 2005.

Mason, G. M., Hamilton, D. C., Walpole, P. H., Heuerman, K. F., James, T. L., Lennard, M. H., and Mazur J. E.: LEICA: A Low Energy Ion Composition Analyzer for the study of Solar and Magnetospheric Ions, IEEE T. Geosci. Remote, 31, 549$556,1993$.

Millan, R. M. and Thorne, R. M.: Review of radiation belt relativistic electron losses, J. Atmos. Sol.-Terr. Phy., 69, 362-377, 2007.

Nicolls, M. J., Kelley, M. C., Vlasov, M. N., Sahai, Y., Chau, J. L., Hysell, D. L., Fagundes, P. R., et al.: Observations and modeling of post-midnight uplifts near the magnetic equator, Ann. Geophys., 24, 1317-1331, 2006, http://www.ann-geophys.net/24/1317/2006/.

Oyama, K. I., Lakshmi, D. R., Kutiev, I., and Abdu, M. A.: Low latitude $\mathrm{Ne}$ and $\mathrm{Te}$ variations at $600 \mathrm{~km}$ during 1 March 1982 storm from HINOTORI satellite, Earth Planets Space, 57, 871878, 2005.

Panasyuk, M. I., Kuznetsov, S. N., Lazutin, L. L., Avdyushin, S. I., Alexeev, I. I., Ammosov, P. P., Antonova, A. E., et al.: Magnetic 
Storms in October 2003, Cosmic Res., 42, 489-534, 2004.

Ridley, A. J., Richmond, A. D., Gombosi, T. I., De Zeeuw, D. L., and Clauer, C. R.: Ionospheric control of the magnetospheric configuration: Thermospheric neutral winds, J. Geophys. Res., 108, 1328, doi:10.1029/2002JA009464, 2003.

Rodger, C. J., Clilverd, M. A., Ulich, Th., Verronen, P. T., Turunen, E., and Thomson, N. R.: The atmospheric implications of radiation belt remediation, Ann. Geophys., 24, 2025-2041, 2006, http://www.ann-geophys.net/24/2025/2006/.

Tinsley, B. A.: Energetic neutral atom precipitation during magnetic storms: Optical emission, ionization and energy deposition at low and mid latitudes, J. Geophys. Res., 84, 1855-1864, 1979.

Tinsley, B. A., Sahai, Y., Biondi, M. A., and Merriwether Jr., J. W.: Equatorial particle precipitation during magnetic storms and relation to equatorial thermosphere heating, J. Geophys. Res., 93, 270-276, 1988.

Tsurutani, B. T., Mannucci, A. J., Iijima, B., Abdu, M. A., Sobral, J. H. A., Gonzalez, W., Guarnieri, F., et al.: Global dayside ionospheric uplift and enhancement associated with interplanetary electric fields, J. Geophys. Res., 109, A08302, doi:10.1029/2003JA010342, 2004.

Tsurutani, B. T., Verkhoglyadova, O. P., Mannucci, A. J., Araki, T., Sato, A., Tsuda, T., and Yumoto, K.: Oxygen ion uplift and satellite drag effects during the 30 October 2003 daytime superfountain event, Ann. Geophys., 25, 569-574, 2007,

http://www.ann-geophys.net/25/569/2007/.
Unnikrishnan, K., Saito, A., Otsuka, Y., Yamamoto, M., and Fukao, S.: Transition region of TEC enhancement phenomena during geomagnetically disturbed periods at mid-latitudes, Ann. Geophys., 23, 3439-3450, 2005, http://www.ann-geophys.net/23/3439/2005/.

Wang, H., Luhr, H., Ma, S. Y., Weygand, J., Skoug, R. M., and Yin, F.: Field-aligned currents observed by CHAMP during the intense 2003 geomagnetic storm events, Ann. Geophys., 24, 311324, 2006, http://www.ann-geophys.net/24/311/2006/.

Yeh, H. C., Su, S. Y., Yeh, Y. C., Wu, J. M., Heelis, R. A., and Holt, B. J.: Scientific Mission of the IPEI Payload Onboard ROCSAT1, Terr. Atmos. Ocean Sci., Supplementary Issue, 19-42, March 1999.

Yizengaw, E., Dyson, P. L., Essex, E. A., and Moldwin, M. B.: Ionosphere dynamics over the Southern Hemisphere during the 31 March 2001 severe magnetic storm using multi-instrument measurement data, Ann. Geophys., 23, 707-721, 2005, http://www.ann-geophys.net/23/707/2005/.

Zhao, B., Wan, W., and Liu, L.: Responses of equatorial anomaly to the October-November 2003 superstorms, Ann. Geophys., 23, 693-706, 2005,

http://www.ann-geophys.net/23/693/2005/. 